\title{
¿Interlocutoras del Estado? El caso de las destinatarias de programas sociales y las referentes de organizaciones territoriales en la Argentina ${ }^{1}$ Interlocutors of the State? The Case of Beneficiaries of Social Welfare and of Representatives of Territorial Organizations in Argentina
}

Carla Zibecchi ${ }^{2}$

\section{Resumen}

El trabajo se propone dar cuenta de las relaciones que establecen las mujeres pobres con el Estado y sus burocracias a través de una descripción de dos casos. El primero se centra en las mujeres como receptoras de los programas sociales asistenciales y el segundo en las representantes de organizaciones territoriales que se desarrollaron en el campo popular en la Argentina. Como uno de sus principales hallazgos se destaca que las mujeres median continuamente entre la población destinataria de la política social asistencial, el Estado y sus agencias, generando nuevas formas de sociabilidad, resolviendo trámites sociales y colaborando a la formación local del Estado.

Palabras clave: Mujeres; Política social asistencial; Burocracias de la calle; Estado; Mediación

\footnotetext{
${ }^{1}$ El trabajo que aquí se presenta forma parte del proyecto: Políticas sociales, campo de poder y actores locales: mujeres beneficiarias, organizaciones territoriales y burócratas de la calle como mediadores de los programas sociales, llevada adelante por la autora en el marco de su labor de investigación para el Consejo Nacional de Investigaciones Científicas y Técnicas (CONICET) de Argentina durante el período 2016-2018.

${ }^{2}$ Doctora en Ciencias Sociales de la Universidad de Buenos Aires (UBA), Argentina. Investigadora del Consejo Nacional de Investigaciones Científicas y Técnicas (CONICET) y del Centro de Investigaciones en Políticas Sociales Urbanas de la Universidad Tres de Febrero (CEIPSU- UNTREF). Profesora Adjunta de la Universidad de Buenos Aires. Buenos Aires, Argentina. Correo electrónico: czibecchi@untref.edu.ar.
}

Universidad del Valle. Cali, Colombia. Recibido: 31/07/2018 Aprobado: 09/11/2018 ISSN: 0122-1213 ISSN-e: 2389-993X Doi: 10.25100/prts.v0i27.7269 


\begin{abstract}
This paper aims to describe the relationships established by poor women with the State and its bureaucracies through a description of two cases. The first case focuses on women as recipients of social welfare programs and the second on representatives of territorial organizations that developed in the popular classes in Argentina. One of the principal findings was that women continuously mediate among the target population of the social welfare policy, the State and its agencies, generating new forms of sociability, resolving social procedures and collaborating with the local formation of the State.

Keywords: women; social welfare policy; street bureaucracies; State; mediation

Sumario: 1. Introducción; 2. Algunos antecedentes significativos del tema y herramientas teóricas; 3. Aspectos Metodológicos; 4. Hallazgos de la investigación: identificando acciones de mediación; 4.1. Las beneficiarias de los PTC: atender la ventanilla, hacer promoción en salud; 4.2 Referentes y cuidadoras comunitarias: la búsqueda de turnos y vacantes; 5. Conclusiones; 6. Referencias bibliográficas.
\end{abstract}

\title{
1. Introducción
}

En la Argentina, las mujeres de sectores populares se desempeñan como coordinadoras de las organizaciones comunitarias con una fuerte inscripción territorial, referentes en sus barrios, beneficiarias de programas sociales asistenciales, entre otros papeles. A través de esta labor cotidiana las mujeres recortan -en un territorio determinado-un campo de actuación que se delimita por una problemática específica (por ejemplo, entregar mercadería a las familias, conseguir un turno en un centro de salud, cuidar niños y niñas del barrio), una población con la cual trabajan de manera cotidiana (los niños, las niñas, sus familias) y un territorio que es definido geográficamente y simbólicamente. En el desarrollo de esta labor diaria, las mujeres son portadoras de un conocimiento específico del territorio, sus 
poblaciones, sus demandas y necesidades, al mismo tiempo que articulan con diversas agencias del Estado y sus burocracias.

En este trabajo nos proponemos describir algunos de los rasgos esenciales que asume esta tarea examinando cómo las mujeres de sectores populares median entre poblaciones específicas y las agencias locales del Estado y sus burocracias. De alguna manera, la hipótesis que nos guía es que, a través de esta labor cotidiana, se transforman en mediadoras e interlocutoras de las burocracias estatales -asistenciales-, especialmente de las "burocracias de la calle".

En función de tal propósito, en primer lugar, recuperamos una serie de trabajos académicos que nos brindan algunas herramientas teóricas y conceptuales. En las últimas décadas se ha producido un importante número de estudios que se preguntan -desde diversos horizontes epistemológicos y posturas disciplinares- en torno a la relación de las mujeres con la política social asistencial, en particular bajo la forma de programas sociales asistenciales (alimentarios, de empleo transitorio, de transferencia de ingresos condicionados). Estos trabajos no sólo constituyen aportes de carácter empírico sino que han contribuido a renovar las preguntas de investigación, a revisar categorías y conceptualizaciones, a proponer estrategias metodológicas y a cuestionar -al mismo tiempo- lecturas androcéntricas y estado céntricas en torno a las relaciones que establecen las mujeres pobres con el Estado (Paura y Zibecchi, 2017).

En segundo lugar, nos centraremos en reconocer algunos rasgos esenciales de las relaciones que establecen estas mujeres con el Estado y sus burocracias a través de la descripción de dos casos que son nuestro

${ }^{3}$ Según Lipsky (1980), los "burócratas de calle" (maestros, policías, jueces, trabajadores sociales, trabajadores de la salud) ocupan un lugar decisivo en la re-hechura de las políticas públicas y su tarea se caracteriza por la interacción cotidiana "cara a cara" con los gobernados, la alta discrecionalidad en aspectos importantes de la implementación de políticas, la relativa autonomía respecto de las autoridades de la organización y las expectativas de una actitud de apoyo activo hacia los gobernados, más allá del contenido específico de las políticas o de los atributos extra-organizacionales de los agentes. En este artículo haremos uso de este término para englobar bajo este rótulo al subconjunto de una agencia pública o institución gubernamental donde trabajan técnicos y profesionales que tienen contacto directo con miembros del público en general (técnicos municipales, maestros, médicos, entre otros). 
principal interés empírico: (i) como receptoras de los programas sociales asistenciales que se implementaron en los últimos años, destacando las prácticas que despliegan en distintos espacios de interacción; (ii) como cuidadoras y referentes de organizaciones territoriales comunitarias que se desarrollaron en el campo popular en la Argentina.

\section{Algunos antecedentes significativos del tema y herramientas teóricas}

En primer lugar, resulta preciso destacar que las nuevas interpretaciones sobre el quehacer cotidiano de las mujeres pobres en sus territorios se enmarcan en la confluencia de perspectivas disciplinares y estrategias metodológicas diversas que habilitaron lecturas microscópicas de las áreas estatales, que abrieron preguntas y agendas de investigación que contribuyeron a dar cuenta de la complejidad del Estado y los estados y, en particular, sobre los procesos de implementación de los programas sociales asistenciales discutiendo miradas estado céntricas (Paura y Zibecchi, $2014)^{4}$. Así, en el reconocimiento de la heterogeneidad y complejidad del Estado se insinuó la presencia de los múltiples actores estatales, la diversidad de los roles y de las representaciones y los mapas cognitivos de los funcionarios de diferente jerarquía (entre ellos: los "burócratas de la calle" $)^{5}$. El aporte de renovadas interpretaciones sobre el estado (nacional), los estados (provinciales, municipales) y sus burocracias en las cuales se

\footnotetext{
${ }^{4}$ Como ya destacamos en otro trabajo (Paura y Zibecchi, 2014), es sabido que no puede obviarse una distinción entre los individuos o grupos de la sociedad como actores sociales y el Estado y sus representantes en distintos niveles, en la medida en que estos gozan de una autoridad legal y de facultades y atribuciones suficientes como para imponer su decisión y forzar las reglas del juego; y al mismo tiempo, los funcionarios y las áreas estatales, -en tanto actores- tienen sus propios intereses. Sin embargo, considerar al Estado como actor -traer al Estado de regreso, en palabras de Theda Skocpol (1985) - posibilita, entre otras virtudes, reconocer la complejidad de su composición heterogénea y sus contradicciones internas. Ya en un trabajo "fundante" en Argentina, Oscar Oszlak (1984) había llamado la atención sobre el carácter contradictorio del funcionamiento del aparato estatal, en tanto arena de conflicto político, como un rasgo constitutivo, antes que como una 'incongruencia' o 'patología'.

${ }^{5}$ En el sentido en que los ha definido Lipsky (1980), los "burócratas de calle" ocupan un lugar decisivo no sólo en la re-hechura de las políticas públicas sino también en la definición de la experiencia de gobierno.
} 
superan abordajes dicotómicos -entre lo micro y lo macro-, han marcado el modo en que se investigan las políticas sociales en tanto permiten reconocer la diversidad de escenarios con fronteras difusas, en donde los actores estatales y no estatales operan y se relacionan. Asimismo, estas interpretaciones implican ver los márgenes del Estado:

"[...] sitios en donde la naturaleza puede ser imaginada como salvaje y descontrolada y donde el estado está constantemente redefiniendo sus modos de gobernar y de legislar. Estos sitios no son meramente territoriales: son también (y quizás sea éste su aspecto más importante) sitios de práctica en los que la ley y otras prácticas estatales son colonizadas mediante otras formas de regulación que emanan de las necesidades apremiantes de las poblaciones, con el fin de asegurar la supervivencia política y económica" (Das y Poole, 2008, p. 24).

Estas nuevas lecturas sobre el estado y los estados y la discusión en torno a las miradas estado céntricas, dieron lugar también al reconocimiento de la heterogeneidad y complejidad de las agencias y escenarios. En el marco de las lecturas microscópicas de áreas estatales, algunos estudios nos alertan sobre la necesidad de situar las investigaciones sobre el estado -en términos conceptuales y empíricos- en sus niveles más capilares y mundanos, en los “encuentros burocráticos" (Gupta, 1995), aparentemente banales.

Siguiendo estas premisas metodológicas es que encontramos relevante el estudio de cómo los programas sociales "se hacen" en diversas escenas, dónde resulta preciso revisar distintos puntos de observación para captar cómo diversos actores median, resignifican y recrean la política social asistencial. En este contexto, la indagación de estas formas de interacción de las mujeres pobres con el Estado y sus burocracias cobra un importante protagonismo.

En segundo lugar, encontramos el desarrollo de otros abordajes en torno al protagonismo de las mujeres en relación con los programas sociales asistenciales, en particular los programas de transferencia de ingresos condicionados (PTC). Estos abordajes innovarán en el estudio de las concepciones implícitas que tienen los PTC en relación con las mujeres, 
en el sentido que gran parte de la profusa literatura sobre la política social asistencial fue realizada desde sesgos androcéntricos en la medida en que no daba cuenta de las concepciones en torno a la mujer, o bien utilizaban categorías con sesgos masculinos en torno al trabajo, invisibilizaban el trabajo de cuidado que realizaban las mujeres e impedían reconocer experiencias disímiles de ellas en relación con el Estado y sus burocracias. En este sentido, numerosos trabajos -algunos de perfil más académico $\mathrm{y}$ otros productos de consultorías con organismos internacionales efectuados por expertas- han comunicado cómo las mujeres desempeñan numerosos "papeles" algunos impuestos por las propias lógicas de diseño de los programas: "beneficiarias", "titulares", "responsables de las condicionalidades", "responsables de las contraprestaciones" (Serrano, 2005; Daeren, 2004; Faur, 2014; Molyneux, 2007; Arriagada y Mathivet, 2007). En particular, destacan que las condicionalidades en salud y educación -como característica central y definitoria de los PTC- tienen importantes implicaciones en la vida de las mujeres: como principales responsables del cuidado de las personas dependientes, en el uso del tiempo que demandan estas tareas (por ejemplo, largas filas para cumplir con la obtención de certificados que deben presentarse para acreditar el cumplimiento de las condicionalidades, los encuentros y charlas educativas a las cuales deben asistir). Sin embargo, esta tarea las ha ubicado en una nueva dimensión ciudadana en tanto funcionan como "interlocutoras" con un Estado "proveedor", poniéndolas en una posición relacional con las instituciones estatales que antes no existía como tal (Rodríguez-Enríquez, $2012)^{6}$. La intensidad y relevancia de estas tareas y la visibilidad que le ha dado, en parte, la implementación de PTC en América Latina ha dado lugar a lo que otros autores llaman el maternalismo en el acceso a los servicios sociales: los servicios sociales estatales se orientan a atender mujeres en

${ }^{6}$ En relación con los cambios en la vida cotidiana de las mujeres que perciben los PTC se destaca: la salida de sus hogares y mayor participación en los espacios comunitarios, las mujeres se sienten más cómodas y confiadas a la hora de hablar en público (Zibecchi, 2013; Daeren, 2004). También se ha constatado que el hecho de que sean las mujeres las que perciben el ingreso, les otorga cierta autonomía financiera con todas las implicancias subjetivas que esto acarrea: elevación de la autoestima, estatus en la comunidad, entre otros importantes efectos (Molyneux, 2007).

Prospectiva. Revista de Trabajo Social e intervención social No. 27, enero-junio 2019: pp. 31-54

(c) (1) $(3)$ 
función de su papel reproductivo o de cuidados (Martínez-Franzoni y Voorend, 2008).

También encontramos otra línea de trabajos que ha demostrado cómo la labor cotidiana de las mujeres se encuentra articulada con las lógicas de poder territoriales y de las organizaciones a través de las cuales desempeñan diferentes roles y funciones: referentes barriales, de organizaciones comunitarias (comedores, guarderías) y de movimientos sociales. De este modo, las mujeres han sido analizadas en su capacidad de liderar y de actuar en territorios determinados. Así, se destaca que las mujeres establecen y recortan un campo de actuación que se va constituyendo en la práctica y que se delimita según las problemáticas específicas y las poblaciones con las que trabajan cotidianamente (Bottaro, 2010; Magariños, 2012). Por su parte, el estudio del lugar particular que desempeñaron las mujeres en las estrategias familiares de sobrevivencia y el abordaje de cómo su trabajo no remunerado es lo que ha absorbido -en gran parte-los costos sociales del ajuste estructural será un aporte sustancial de los estudios de género. En particular, diversos trabajos se centraron en el análisis de las modalidades de participación de las mujeres con especial énfasis en las prácticas alimentarias (ollas populares, comedores, compras comunitarias, distribución de alimentos) (Fernández, 1992). Estudios más recientes han destacado el rol de las mujeres en la construcción de redes alimentarias como una forma de articulación social producida por las mismas mujeres (Garrote, 2003).

Finalmente, desde perspectivas etnográficas se han investigado las interacciones cotidianas en las ventanillas y salas de espera, analizando las relaciones entre agentes y usuarios. Investigaciones centradas en la burocracia asistencial de Francia examinan -desde esta perspectiva- el intenso trabajo relacional que efectúan las mujeres en las ventanillas frente a los profesionales del sector público - para recibir subsidios para viviendas sociales, pensiones, entre otras-. En estos casos los saberes prácticos administrativos de las mujeres pobres, su inversión de tiempo, su trabajo de cuidado son claves para entender cómo se concreta la política social a nivel local y, al mismo tiempo, se constituye en un "telón de fondo" en el cual se mezclan prácticas burocráticas e interpelaciones para alcanzar derechos y protecciones (Schijman y François-Laé, 2011). Desde 
un abordaje etnográfico similar pero centrándose en el caso argentino, se ha estudiado a las mujeres que "peregrinan" por los pasillos de la burocracia estatal (Auyero, 2013).

Retomando los aportes de estas investigaciones nos interesa abordar las relaciones que establecen estas mujeres con el Estado y sus burocracias asistenciales. Nuestro principal interés empírico se circunscribe en este artículo a dos casos -que serán desarrollados a continuación- cuyo estudio consideramos central como insumo para comprender cómo se concreta la política social en un sentido amplio (programas sociales, PTC, políticas sectoriales como salud y educación) a nivel local.

\section{Aspectos metodológicos}

En los apartados que siguen se desarrollan dos casos. El primero se centra en las mujeres como receptoras de los PTC que se implementaron en los últimos años, destacando las prácticas que despliegan en los espacios de interacción; y el segundo en las mujeres como cuidadoras y referentes de organizaciones territoriales que se desarrollaron en el campo popular en la Argentina. Consideramos que ambos casos pueden resultar claves para el estudio de las mujeres en su interacción con el Estado -sus instituciones y sus burocracias- y esto obedece a una serie de criterios epistemológicos y metodológicos que delimitan el enfoque elegido.

- La relevancia de poner el foco empírico en las prácticas relacionales de las mujeres con el Estado -sus burocracias e instituciones-según sea el caso o situación que se analice. Es decir, nuestro interés en este trabajo no está puesto en el programa en sí (por ejemplo, las características programáticas del PTC) o la organización territorial de la que se trate (un comedor comunitario, una guardería comunitaria), como tampoco lo está en una política específica (salud, educación), sino en una dimensión relacional: las prácticas que permiten mediar, generar reciprocidades y formas de sociabilidad en el mundo asistencial y que llevan adelante las mujeres a través de diversos papeles (receptoras de PTC, referentes de organizaciones territoriales, cuidadoras) y en distintos contextos (una organización territorial, el barrio, la ventanilla de una oficina pública). 
- Considerar que los encuentros cotidianos de estas mujeres con las burocracias estatales le dan forma y marco concreto a lo que sería de otra forma una gran abstracción (El Estado) y, al mismo tiempo, constituyen la construcción cotidiana del Estado (Gupta, 1995). En este sentido, nos interesa ubicarnos en la tensión entre no personalizar al Estado - en tanto se plantea considerarlo como un espacio polifónico en el cual se relacionan y se expresan diversos grupos- pero, al mismo tiempo, la relevancia de personalizar al Estado en cuanto son las personas que lo producen y lo actualizan con sus prácticas cotidianas (Bohoslavsky y Soprano, 2010).

- Problematizar no solo las relaciones de clase que establece el Estado sino también las relaciones de género, en tanto las burocracias emplean símbolos culturales para relacionarse con la población que recibe ayuda social y establecen criterios acerca de las personas que son o no merecedores de asistencia. Como veremos a continuación, es el mismo Estado quien coloca en esta posición relacional a las mujeres cuando, por ejemplo, les solicita como condición para cumplir con un PTC que atienda una ventanilla de una oficina pública porque considera que reúne ciertos atributos para hacerlo (entre otros: ser mujer, madre). Según destaca Ana Laura RodríguezGustá (2014), algunas corrientes dentro de las teorías feministas del Estado han examinado las interacciones entre actores públicos y poblaciones destinatarias de los programas sociales. Según la autora:

Ello ocurre, primordialmente, porque las burocracias responsables por las intervenciones sociales no son meras poleas de transmisión de construcciones culturales sino que tienen interpretaciones respecto del trabajo remunerado, del papel normativamente asignado a mujeres y hombres y del valor de la reproducción social (actividades de cuidado y domésticas). Desde este ángulo, las creencias y las representaciones de los funcionarios públicos (definidos en un sentido amplio) influyen en las orientaciones de las políticas al incidir en las acciones micro que hacen a la implementación (Goodwin, 1997 citado en Rodríguez-Gustá, 2014, p. 561). 
- A un nivel empírico, entonces, podemos pensar que estas burocracias interactúan cotidianamente con mujeres, en tanto son mayoría en la población pobre, son las principales demandantes de subsidios y servicios estatales, las principales encargadas el cuidado de sus familias y conocedoras de sus necesidades en relación con los servicios sociales, las responsables de cumplir con las condicionalidades y contraprestaciones que imponen los PTC, para nombrar algunos aspectos que explican el hecho que los encuentros cotidianos del Estado con los pobres tiene una dimensión de género que ocupa un lugar central en el análisis?

Como veremos en los casos que se describen a continuación, los espacios de indagación son diversos. En efecto, en esta relación entablada entre las mujeres y el Estado -a través de los programas- los escenarios en los cuales se centrarán los análisis podrán ser los lugares donde efectúan las contraprestaciones (organizaciones territoriales, los municipios), donde se cumplen las condicionalidades vinculadas a la certificación de la salud y la escolaridad de los hijos/as (los centros de salud, las escuelas), las propias oficinas vinculadas con la burocracia asistencial (las ventanillas) donde interactuarán cara a cara con los "burócratas de la calle", entre otros. En ese sentido, tanto la realización de entrevistas en profundidad como de observaciones permiten habilitar estas lecturas y hacer inteligibles fenómenos antes invisibilizados. Los casos se construyeron a través de los hallazgos empíricos de dos trabajos de campo. En primer lugar, se presentan algunos de los resultados provenientes de entrevistas a mujeres beneficiarias de PTC (en particular del Plan Jefes y Jefas de Hogar y el Programa Familias) que efectuaban su contraprestación en diversas organizaciones territoriales y en instituciones estatales (oficinas municipales, escuelas, centros de salud) ubicadas en la Región

\footnotetext{
${ }^{7}$ Como destaca Marques-Pereira (1993) si bien no se puede hablar de la existencia de una teoría feminista del Estado, está claro que la reflexión metodológica del enfoque del Estado a partir de las relaciones de género constituye hoy una postura teórica importante. Como destaca el autor, los estudios más recientes del Estado benefactor y las mujeres demuestra que este no es monolítico ni todopoderoso frente a la evolución de las relaciones de poder, rechazando la reducción de las relaciones de género sólo a las condiciones materiales de producción y considerando a estas relaciones en sus dimensiones sociales y políticas.
}

Prospectiva. Revista de Trabajo Social e intervención social No. 27, enero-junio 2019: pp. 31-54 
Metropolitana de Buenos Aires (RMBA) de la Argentina, en particular en zonas de alta concentración de pobreza e indigencia. En relación con las cuidadoras y referentes de organizaciones territoriales se efectuaron entrevistas a mujeres en villas y barrios periféricos de la RMBA que desempeñaban tareas de conducción y cuidado -en calidad de referentes, cuidadoras- en organizaciones territoriales que cuidaban niños y niñas en contextos de pobreza (guarderías, jardines comunitarios). ${ }^{8}$

\section{Hallazgos de la investigación: identificando acciones de mediación}

\subsection{Las beneficiarias de los PTC: atender la ventanilla, hacer promoción en salud}

Bajo la finalidad de comprender el contexto de esta acción de mediación resulta importante destacar que la crisis de $2001-2003^{9}$ marca un punto de inflexión en las formas de intervenir frente a la pobreza del Estado argentino. A partir de este período, comienzan a implementarse los PTC cuyo caso más emblemático ha sido el Plan Jefes y Jefas de Hogar (PJJHD) que tuvo un componente de actividades comunitarias -al igual que los programas de empleo transitorio que lo precedieron- y si bien no contempló un cupo para mujeres, entre sus filas de receptores reunió a un alto porcentaje. Se trataba de una transferencia monetaria de suma fija, destinada a jefes o jefas de hogar desocupados con hijos de hasta 18 años de edad o discapacitados de cualquier edad, o a hogares en los que la jefa de hogar o la cónyuge, concubina o cohabitante del jefe estuviera en estado de gravidez.

${ }^{8}$ En relación con los PTC las entrevistas y observaciones fueron realizadas durante el período 2003-2009 que fue el momento en el cual en la Argentina se implementaron los PTC -el Plan Jefes y Jefas de Hogar se implementó desde el año 2002 y el Programas Familias desde el 2006- ya que a partir del año 2009 los mismos fueron reemplazados por la Asignación Universal por Hijo para la Inclusión Social. En relación con las entrevistas a las referentes y cuidadoras de las organizaciones territoriales, las mismas se efectuaron durante el período 2010-2014.

${ }^{9}$ Se trató de la crisis institucional, política y económica más importante de la historia argentina luego de una recesión económica de casi 5 años, con un deterioro profundo de los principales indicadores sociales.

Prospectiva. Revista de Trabajo Social e intervención social No. 27, enero-junio 2019: pp. 31-54 
En este contexto, veremos a continuación el caso de Paulina, una joven receptora del PJJHD en tanto resulta significativa su experiencia y permite observar cómo las mujeres receptoras colaboraron con los técnicos municipales inscribiendo a otros receptores, recibiendo la documentación, atendiendo consultas y demandas, siendo la "cara visible" del PJJHD en las ventanillas del Estado en el contexto de la crisis 2001-2003. Atendiendo a cierta lógica de reclutamiento de las mujeres beneficiarias por parte de las "burocracias de la calle" que pueden colaborar en la tarea diaria y cotidiana del Estado, la misma se basa en ciertos atributos y cualidades de las cuales ellas son portadoras (mujeres, madres) y otros como el nivel educativo alcanzado -Paulina ya había culminado los estudios secundarios y comenzaba a transitar sus primeros pasos por la universidad-, sus deseos imperiosos por cumplir con la contraprestación, entre otros aspectos. ${ }^{10}$

Así, a partir de los primeros días del lanzamiento del plan, como receptora del PJJHD es convocada a sumarse para inscribir a otros beneficiarios y, de esa manera, realizar la contraprestación requerida. Tal primera experiencia es recordada como muy dolorosa por las historias que escuchaba de los potenciales receptores y por la imposibilidad de poder seguir inscribiendo luego de la fecha límite del cierre del plan. Paulina que, como tantas otras personas, nada sabía acerca de la fecha límite para la inscripción, contó haber sentido mucha culpa cuando atendió a un hombre y le dijo que vuelva la semana siguiente, porque le faltaba un papel. De hecho, cuando los vecinos del barrio se enteraron que Paulina "inscribía para el Jefes" pronto fueron a buscarla:

Y las historias de vida que escuchaba... cada cosa jeran tristes! Aparte yo conocía un montón de beneficiarios porque trabajaba en la zona donde vivía... Al lado de mi casa había un hotel, ¡cuando se enteraron que yo inscribía, te imaginás que fueron todos a buscarme...!

${ }^{10}$ De modo que es preciso también desentrañar los conflictos que esta nueva posición relacional trae consigo en particular vinculados con los criterios de merecimiento que manejan y construyen las mujeres en los territorios en los cuales se encuentran insertas (con las "burocracias de la calle", con las familias "beneficiarias"). En otro trabajo (Zibecchi, 2013) dimos cuenta de cómo las burocracias escolares o sanitarias frecuentemente estigmatizaban a estas mujeres como "las del plan" en su labor diaria.

Prospectiva. Revista de Trabajo Social e intervención social No. 27, enero-junio 2019: pp. 31-54

(c) (i) (3) $\Theta$ 
Terminado el período de inscripción al PJJHD, la siguiente actividad de Paulina como receptora consistió en informar a otras personas asistidas por el plan sobre las fechas de pagos del subsidio, lo que para ella significaba transmitir a la población la información, "poner la cara” y, además, recibir la demanda permanente.

era junio, iy eran los reclamos de la gente que no salía beneficiaria del plan y se había anotado en un primer momento y no salía beneficiada! Eran motivos por desorden administrativo, nosotros juntábamos planillas y poníamos la documentación adentro, y no es un sistema muy seguro... se traspapelaba... aparte las personas encargadas de cargar los datos era gente beneficiaria del plan y algunos eran gente capacitada, pero otros no, no eran gente capacitada, muy alto el nivel de error... y yo tenía que atender a toda esa gente... Porque ellos me hacían poner la cara a mí... [...] yo laburaba un montón: laburaba de lunes a viernes, sabía todo lo de Jefes y Jefas, sabía todo de los beneficiarios, lo que vos me preguntabas, yo lo sabía...

En el marco del PJJHD otras mujeres efectuaron otra modalidad de contraprestaciones, por ejemplo, Lorena se desempeñó como promotora en salud y otras actividades vinculadas a la implementación del PJJHD y el Programa Familias en su barrio. ${ }^{11}$ El testimonio que presentamos a continuación refleja, por un lado, la alta valorización de esta tarea al punto tal de considerarla como un encuentro de la vocación, al mismo tiempo que le permitió sentirse valorizada y reconocida. Por otra parte, muestra la intensa actividad de mediación que realizaba entre el Estado y las poblaciones en territorios delimitados: capacitaciones, actividades de promoción en salud, censos para las familias, entre otras de vital importancia para un Estado que necesita que su población beneficiaria sea objetivable y manejable bajo los imperativos de la focalización. La historia de Lorena y cómo fue "reclutada" para realizar estas tareas también se relaciona con la violencia de género que atraviesa todo su relato. En búsqueda de ayuda -separada

${ }^{11}$ El Programa Familias para la Inclusión Social consistió en la entrega de una transferencia monetaria a familias en situación de pobreza, con hijos/as menores de 19 años, cuya recepción estaba condicionada a la atención del cuidado de la salud de las mujeres embarazadas y los/as niños/as y la permanencia de estos últimos en el sistema educativo, y la promoción familiar y comunitaria, mediante acciones de promoción en términos de educación, salud, capacitación para el trabajo y desarrollo comunitario. 
y con cinco hijos a cargo- y estando desocupada fue atendida por parte de los profesionales del área de acción social del municipio quienes le fueron proponiendo contraprestaciones y condicionalidades a través de los diversos programas sociales que tuvo desde fines de los años noventa (programas de empleo transitorio, PTC, entre otros) ${ }^{12}$.

bueno termina este $[\mathrm{PEL}]^{13}$ y vamos a la capacitación en prevención de enfermedades que nos la dan médicos, enfermeros... nos dan diferentes capacitaciones en distintas temáticas: diabetes, HIV, planificación familiar [...] ¡Esto nos fascinó! ¡Nos hizo sentir que podíamos dar al otro, ayudarlo con una prevención! ¡Y nos fascinó sobre todo la planificación familiar! Porque casi todas las mamás, somos de más de 4 o 5 hijos, entonces, cuando nos hizo el "clic" es como que comprendimos lo de poder buscar un hijo, la felicidad de esperarlo,... muchas cosas que no teníamos en claro por estar viviendo otro momento de nuestras vidas... bueno, nos gustó tanto que empezamos a trabajar fuertemente con esto... trabajábamos como promotoras de salud en la sala, se trabajó mucho con la parte de epidemiología también, y después de esto nosotras elegimos trabajar fuertemente con planificación familiar y en prevención del HIV [...] siempre con el acompañamiento de varias trabajadoras sociales del municipio del área de salud [...] bueno trabajamos un tiempo más en esto y llega el tiempo del Programa Familias [...] me dan la oportunidad de hacer el censo para el Familias, [...] en algunas cosas nos ponían para poder ganar un poco de dinero nosotras como asistentes de talleres, cuando hubo talleres de Familias para las condicionalidades [...] siempre nos dieron oportunidad, siempre nos dieron libertad, me he encontrado con gente maravillosa de verdad, que nos dejó crecer, que creyó en nosotros que eso es lo más importante.

\section{Ahora bien, ¿por qué estas experiencias pueden ser significativas} como puntos de observación? Paulina, se transforma en su barrio en la "cara visible" del Estado en tanto inscribía a otros receptores y atendía sus reclamos, es decir Paulina era para otros receptores/as o vecinos del barrio, que necesitaban ingresar al plan, la presencia del Estado en sus

${ }^{12}$ Para más desarrollo de este tema, ver Zibecchi (2013).

${ }^{13}$ El Programa de Emergencia Laboral (PEL) se trató de un programa de empleo transitorio para personas desocupadas mayores de 18 años y preferentemente jefe o jefa de hogar que proponía obras o servicios a la comunidad. Se implementó en la Argentina durante el período 2000-2002, antes del PJJHD.

Prospectiva. Revista de Trabajo Social e intervención social No. 27, enero-junio 2019: pp. 31-54

(c) (i) (3) $\Theta$ 
vidas cotidianas. Lorena, por su parte, efectuaba capacitaciones y talleres para que otras mujeres pudieran cumplir con las condicionalidades que impone la política asistencial. No menos importante, es su participación censando familias para ingresar a los programas, colaborando en el llenado de formularios, entre otras acciones.

Como puede observarse, a un nivel cotidiano y microsocial, el Estado construye poblaciones objeto (en este caso, familias pobres) parar intervenir (adjudicarles un PTC) y para controlar (a través de imponer requisitos en condicionalidades en salud y educación que deben cumplir las madres pobres). Las mujeres, por su parte, dependen de este Estado en su condición de beneficiarias, donde un atributo central de esta relación reside en su condición de madres y cuidadoras. Al mismo tiempo, acceden a un programa -como dispositivo de intervención y manifestación de la acción estatal- que estructura y da forma a las relaciones de género: las ubica en una nueva posición relacional que les permite interactuar de otro modo con poblaciones y con el Estado y sus agencias, pero al mismo tiempo las somete al cumplimiento y el control de las condicionalidades y contraprestaciones.

A través de estas acciones las mujeres generan nuevas formas de sociabilidad y de mediación entre el mundo público -el Estado y sus programas- y el privado -otras mujeres como ellas, sus familias y los vecinos del barrio-. En otros términos, en los diversos escenarios de interacción (las ventanillas, las organizaciones territoriales), estas mujeres encarnan la figura de lo público y del Estado, con toda la complejidad que reviste que sujetos privados (la receptora del PJJHD, la beneficiaria del Programa Familias) resuelva asuntos de interés público (recepción de demandas, censos, inscripción a programas sociales).

\subsection{Referentes y cuidadoras comunitarias: la búsqueda de turnos y vacantes en el territorio}

La participación de la mujer en las organizaciones territoriales se enmarca en el fenómeno de la feminización del mundo comunitario, definiéndolo a este último como un espacio en el que se cruzan las necesidades de los sectores populares, las políticas sociales implementadas por el Estado a través de las diferentes organizaciones sociales que hacen las veces de 
mediador entre ambos. En dicho mundo, las mujeres ocupan el lugar de "referentes", "beneficiarias", "voluntarias", entre otros roles (Bottaro, 2010). Como consecuencia de dicho fenómeno, se observa que en el caso de las organizaciones territoriales dedicados al cuidado de niños y niñas -guarderías, jardines comunitarios- existe una presencia casi exclusiva de

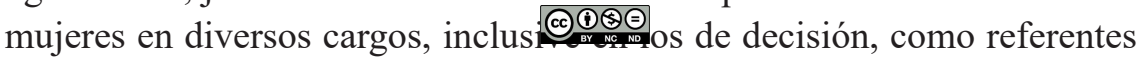
y coordinadoras. De modo que las organizaciones territoriales que proveen cuidado comunitario son espacios protagonizados y dominados por mujeres (Zibecchi, 2015).

El centrarnos en este tipo de organización territorial como espacio de indagación nos brinda un punto de vista estratégico para analizar la mediación que realizan las mujeres entre las burocracias locales y las poblaciones a las que asisten. Esto es así porque gran parte de las políticas y programas sociales asistenciales de los últimos años han sido los principales generadores de recursos - dinero, becas, alimentos, capacitaciones- a las organizaciones que proveen de cuidados comunitarios imprimiendo, al mismo tiempo, dinámicas particulares a las prácticas cotidianas de las mujeres que allí trabajan (Zibecchi, 2015). Al mismo tiempo, este tipo de organización depende en gran medida de diversos servicios e instituciones estatales: asistencia de trabajadoras sociales del estado local -que puedan colaborar en la derivación de casos y necesidades de niños y niñas-, centros de salud y hospitales, escuelas y jardines de infantes oficiales dependientes también de los municipios (Pautassi y Zibecchi, 2010).

En síntesis, al complejo soporte político institucional en el cual se insertan las guarderías y jardines comunitarios se suma el entramado que se construye diariamente -también a través de una labor intensa por parte de las mujeres- en los territorios, lo que le da otra particularidad a este tipo de organizaciones: la forma de trabajo asociada y/o en red. Más allá de que algunas organizaciones conformen una red exclusiva, en la mayoría de los casos se vinculan con otras instituciones del territorio en el cual se encuentran insertos, en particular se relacionan con los centros de salud y sus profesionales y con las escuelas primarias -principalmente, de gestión estatal municipal- a las que asisten los niños y niñas una vez que egresan del jardín. 
Ahora bien, ¿cómo se articulan estas acciones estatales y programas sociales con el trabajo cotidiano de cuidado a nivel local? ¿Cómo se llevan adelante estas lógicas de trabajo articuladas con otras instituciones del territorio? Ciertamente, se presentan tantas situaciones como casos explorados. En efecto, estas organizaciones territoriales presentan apoyos de distintos programas sociales, al mismo tiempo que muestran distintas dinámicas de relaciones con los estados municipales, provinciales y nacionales. Sin embargo, una particularidad que se presenta de manera recurrente es el trabajo de mediación permanente que estas organizaciones realizan entre los estados y las poblaciones a las cuales asisten y, dentro de ellas, el trabajo de las referentes y cuidadoras de estos espacios territoriales ocupa un lugar muy protagónico. Como lo destaca una coordinadora de un jardín comunitario:

Digamos... la red la establecemos bastante nosotros, si bien el gobierno nos da herramientas sobre violencia familiar, violencia de género, cómo funciona una defensoría... nos acercan esa información y hay capacitaciones a lo largo del año o reuniones informativas. También es verdad que cada [organización] arma sus redes en función del territorio, tomando algunas instituciones públicas que brindan determinados servicios y son del barrio $[\ldots]$ nos reunimos una vez por mes, intercambiamos propuestas, en que anda cada uno, sobre todo conocernos, si uno tiene que hacer alguna derivación o alguna consulta podemos darnos una mano.

Si bien la intensa tarea relacional de mediación de las mujeres puede observarse a través de los diversos programas y dispositivos estatales programas alimentarios, becas estudiantiles, subsidios a las organizaciones territoriales- a continuación se describen dos situaciones en las cuales las mujeres son permanentemente mediadoras, esto es, el acceso al sistema educativo (nivel inicial estatal) y el sistema sanitario.

En efecto, dependiendo de los casos y del nivel de cobertura que tengan (de salitas o grados según grupo etario), algunos niños/as deberán asistir al preescolar o a primer grado de la escuela primaria obligatoria ${ }^{14}$. En este

${ }^{14}$ La educación inicial en Argentina comprende a los/as niños/as desde los cuarenta y cinco (45) días hasta los cinco (5) años de edad inclusive, siendo obligatorio los dos últimos años.

Prospectiva. Revista de Trabajo Social e intervención social No. 27, enero-junio 2019: pp. 31-54 
contexto, el vínculo establecido por las mujeres (generalmente, referentes o coordinadoras de las organizaciones) con los jardines de infantes estatales se torna imprescindible. Dado el escaso nivel de institucionalización bajo la cual muchas de las organizaciones efectúan tareas educativas y de cuidado, frecuentemente no cuentan con los medios para extender certificados y credenciales que acrediten efectivamente los de los años de asistencia, los saberes y aprendizajes de los niños/as. Allí radica, entonces, la importancia de ciertos actores que puedan recomendar, reservar vacantes y establecer relaciones con otras instituciones del Estado a nivel local. Impulsadas por los requisitos impuestos por escuelas de la zona, algunas coordinadoras y referentes de organizaciones territoriales desarrollaron estrategias con vistas a poder extender un certificado o constancia que demuestre, precisamente, que los y las niñas transitaron por esas organizaciones como parte de su formación. Asimismo, similares estrategias fueron implementadas para lograr tener la Clave Única de Establecimientos ${ }^{15}$ y que las familias puedan rendir el cumplimiento de las condicionalidades.

En este contexto, entonces, una recomendación, un buen contacto a través de una maestra, que una organización o jardín tenga "buena fama", un llamado por teléfono para reservar una vacante, se torna un medio central - estratégico- para que estos niños y niñas puedan acceder a instituciones estatales (por ejemplo, al preescolar en un contexto de escasa oferta pública).

En relación con el sector salud, estas mujeres establecen relaciones con las salas de primeros auxilios, centros de salud comunitarios u hospitales del barrio en situaciones y bajo formas bien precisas: (i) cuando un niño/a se lastima o se descompone, asisten inmediatamente o llaman a la ambulancia ; (ii) campañas de vacunación y prevención, a partir de las cuales muchas veces los profesionales de los centros de salud se acercan a las organizaciones territoriales; (iii) contactos con profesionales (asistentes sociales, pediatras, psicólogos) de las unidades sanitarias para resolver controles médicos cotidianos (en especial, de peso y de alimentación) y

${ }^{15}$ El CUE es un número que identifica a cada una de las escuelas de la Argentina. Algunos jardines comunitarios ya lo han logrado y los niños que allí asisten pueden acreditar la asistencia escolar de la misma forma que si asistieran a un jardín público de gestión estatal. No obstante, no todas las organizaciones lo han logrado, está en continuo proceso esta acreditación siendo, por cierto, un motivo de lucha de los diversos jardines. 
problemáticas específicas (niños/as que han sufrido violencia y maltrato físico, etc); (iv) de modo frecuente son las cuidadoras educadoras las que solicitan certificados de salud o de vacunación y envían a los padres a la sala para tener la documentación al día, del mismo modo lo hacen si detectan un niño/a enfermo; (v) se observa que cuando se efectuaron capacitaciones, éstas fueron originadas por la propia iniciativa de las mujeres referentes y cuidadoras de las organizaciones que tienen acceso a la información y a contactos personales con especialistas y profesionales que efectúan ciclos de charlas y capacitaciones. Cualquiera sea el caso, el trabajo de acompañamiento, atención, búsqueda de turnos, controles de vacunación lo realizan ellas:

... Nosotros tenemos la salita a cuatro cuadras, entonces, con la autorización de los padres, los llevábamos a un control médico. El pediatra nos esperaba, cuando terminaba de atender a todos sus pacientes, nos atendía a todos los nenes. Íbamos con el carnet de vacunación con la autorización del padre y los vacunábamos, les hacíamos un control y, bueno, de ahí nos derivaba si necesitaba hierro, algún estudio ya eso se encargaban los padres... Ya después de hacer esto muchos años de llevarlos, traerlos, un día nos llama el pediatra y nos dice: "No vengan, que vamos a ir nosotros ¿eh?". Así que vino el pediatra y las enfermeras y le hicieron un control a todos los nenes... (Coordinadora de un jardín comunitario)

... tenemos una pediatra que es hija de una chica de la Cooperativa, que también viene a hacer los controles, no siempre. Pero una vez por mes viene y mira toda la vacunación que esté todo en orden... Viene porque la mamá trabajó mucho tiempo con nosotros, ahora está trabajando en fábrica y conoce la labor nuestra, entonces, ella por amor que nos tiene... (Coordinadora de un jardín comunitario que depende de una cooperativa de trabajo).

Las referentes y coordinadoras de las organizaciones median permanentemente entre el Estado y sus burocracias (escolares, sanitarias, asistenciales) y las poblaciones más necesitadas a través de la negociación permanente, con reglas formales e informales, para acceder a diversos recursos, servicios, pero sobre todo a derechos. Así, se produce una labor intensa relacional de las mujeres de las organizaciones con las burocracias del Estado, en particular con los "burócratas de la calle" y que conlleva 
recorridos por diversas instituciones y oficinas del Estado local en búsqueda de vacantes, prestaciones, servicios, derivaciones, turnos.

\section{Conclusiones}

Las premisas teóricas y metodológicas planteadas al comienzo fueron la hoja de ruta que permitió hacer una lectura de los dos casos empíricos presentados, habilitó un acercamiento a la participación de las mujeres de sectores populares en lo social que se aparta necesariamente de una mirada que las ubica en un lugar desinteresado, altruista -que participan solo por voluntad, por gratitud-, manipuladas por relaciones clientelares o subsumidas por la relación de opresión y dominación que les imprime el Estado. Por el contrario, la propuesta fue empezar a entender esta problemática desde otra perspectiva: las mujeres como protagonistas y constructoras de un entramado de relaciones con el Estado, sus burocracias e instituciones a través de las cuales desarrollan prácticas, saberes y mediaciones específicas. Por otra parte, los casos elegidos permitieron hacer inteligible esta labor diaria y delinear algunos de sus principales rasgos que a continuación se enumeran.

En primer lugar, observamos que las mujeres analizadas (beneficiarias del PTC y/o referentes de las organizaciones territoriales) no forman parte de la estructura del Estado, no forman parte de él en un sentido estricto del término: no son agentes oficiales, tampoco trabajadoras estatales, no forman parte de la estructura burocrática. Sin embargo, estas mujeres aparecen como referentes del espacio público y recrean expresiones de lo público y de lo estatal.

En segundo término, en el cumplimiento de estas funciones median continuamente entre la población destinataria de la política social asistencial (mujeres pobres y madres, como ellas) y diversos "burócratas de la calle". Es decir, las mujeres, en calidad de mediadoras, tienen relación cara a cara con maestros, médicos, trabajadores sociales, burócratas asistenciales, entre otros, y las poblaciones destinatarias generando nuevas formas de sociabilidad, son agentes que pueden tramitar problemas sociales y colaboran a la formación local del Estado. 
En tercer lugar, a un nivel más específico, en el primer caso revisado advertimos que las mujeres receptoras de PTC son mediadoras entre estos dispositivos de intervención estatal y otras poblaciones, que al igual que ellas son potencialmente destinatarias de estos programas y demandantes de los mismos. Las mujeres receptoras de los PTC desempeñan numerosas funciones como lo destacan las investigaciones del tema que en este artículo hemos mencionado. La lista es amplia y extensa: acreditar el cumplimiento de las condicionalidades, cumplir con las contraprestaciones laborales que impone el propio diseño de los programas, convertirse en promotoras y organizar encuentros y charlas para que otras mujeres -beneficiarias, madres y pobres- "cumplan" con lo propio, realizar reclamos en las ventanillas para cobrar el subsidio, atender reclamos, efectuar las colas, recorrer las oficinas de los servicios sociales, esperar respuestas.

En cuarto término, en el segundo caso se pudo constatar que las mujeres cuidadoras y/o coordinadoras de las organizaciones recortan en un territorio determinado, un campo de actuación que se delimita por una problemática específica (el cuidado que puede proveer la comunidad), una población con la cual trabajan de manera cotidiana (los niños, niñas y sus familias) y un territorio que es definido geográficamente y simbólicamente (ellas son del barrio, conocen los problemas de la gente, de las familias). A su vez, son portadoras de un conocimiento específico del territorio (vecinos que pueden colaborar, infraestructura sanitaria y escolar del territorio, burocracias con las cuales deben lidiar de manera diaria), al mismo tiempo que articulan con diversas instancias del Estado local (escuelas, salas de primeros auxilios, centros de salud).

Como ya destacamos, los saberes prácticos administrativos de las mujeres pobres, su inversión de tiempo, su trabajo de cuidado son claves para entender cómo se concreta la política social a nivel local. Pero además es importante entender que se trata de actividades cotidianas, subrepticias silenciosas pero constantes (Schijman y François-Laé, 2011). Asumiendo el riesgo de señalar algunas cuestiones recurrentes, se puede destacar que las mujeres realizan las siguientes prácticas que conllevan saberes específicos: organizando y dictando talleres de prevención y de cuidado a las familias, realizando llamados por teléfono o haciendo largas filas, acompañando a 
las familias a las instituciones, asignando becas, estableciendo vacantes y conformando poblaciones a atender a través de las organizaciones que lideran, realizando denuncias por violencia de género en las comisarías de la mujer, distribuyendo comida (a través del comedor, a través de viandas), completando formularios, organizando legajos, y la lista continúa. A través de esta intensa tarea aprenden un lenguaje específico, conocen procedimientos administrativos, burocráticos y judiciales, reconocen el valor de los documentos, aprenden a organizar legajos, manejan relaciones cara a cara con los profesionales del sector público, llevan adelante una propia contabilidad y administración de los recursos que llegan a la organización y que deben ser distribuidos.

Finalmente, estos casos permiten revisar nuevas teorías acerca del Estado $^{16}$ que nos alertan, no sin razón, que ya no es posible tratar a la naturaleza opresiva del Estado sobre las mujeres a priori de modo tal que no permite captar cómo las mujeres han obtenido nuevas posiciones -en este caso, como mediadoras, como interlocutoras- con una fuerte inscripción en sus subjetividades -posicionarse como referentes de una organización, ser reconocidas por los otros, encontrar una vocación, ser la cara visible de un programa, de una organización en el barrio-. Al mismo tiempo, no subestimar el ejercicio de control social del Estado sobre las mujeres: que deben cumplir con las contraprestaciones y condicionalidades y, además, hacer que otras mujeres lo cumplan.

\section{Referencias bibliográficas}

Arriagada, I. y Mathivet, Ch. (2007). Los programas de alivio a la pobreza Puente y Oportunidades. Una mirada desde los actores. Chile: CEPAL.

Auyero, J. (2013). Pacientes del Estado. Argentina: Eudeba.

Bohoslavsky, E.L. y Soprano, G. (Eds.). (2010). Un Estado con rostro humano.

Funcionarios e instituciones estatales en Argentina (desde 1880 hasta la actualidad). Argentina: Prometeo-UNGS.

Bottaro, L. (2010). Organizaciones sociales, representaciones del trabajo y universo femenino en el espacio comunitario. En G. Kessler, M. Svampa, I. González-Bombal, I. (Comps.) Reconfiguraciones del mundo popular. El

${ }^{16} \mathrm{Al}$ respecto se puede ver la revisión que efectúa Marques-Pereira (1993). 
conurbano bonaerense en la postconvertibilidad (pp. 125-178). Buenos Aires: UNGS/Prometeo.

Daeren, L. (agosto, 2004). Mujeres Pobres: Prestadoras de servicios o sujetos de derecho? Los programas de superación de la pobreza en América Latina desde una mirada de género. En Seminario Internacional Políticas y programas de superación de la pobreza desde la perspectiva de la gobernabilidad democrática y el género. Quito, Ecuador.

Das, V. y Poole, D. (2008). El estado y sus márgenes. Etnografías comparadas. Cuadernos de Antropología Social, (27), 19-52.

Faur, E. (2014). El cuidado infantil en el Siglo XXI: mujeres malabaristas en una sociedad desigual. Argentina: Siglo Veintiuno Editores.

Fernández, A.M (Comp.). (1992). Las mujeres en la Imaginación Colectiva: Una historia de Discriminación y Resistencia. Argentina: Paidós.

Garrote, N. (2003). Redes alimentarias y nutrición infantil. Una reflexión acerca de la construcción de poder de las mujeres a través de las redes sociales y la protección nutricional de niños pequeños. Cuadernos de Antropología Social, (17), 117-137.

Gupta, A. (1995). Blurred Boundaries: The Discourse of Corruption, the Culture of Politics, and the Imagined State. American Ethnologists, 22(2), 375-402.

Lipsky, M. (1980). Street-Level Bureaucracy: dilemmas of the Individual in Public Services. New York: Russell Sage.

Magariños, P. (2012). Líderes localizadas: perfiles de mujeres actuando en territorios. En N. Gherardi (Dir.) Lidera: participación en Democracia. Experiencias de mujeres en el ámbito social y político en la Argentina (pp.115127), Argentina: Equipo Latinoamericano de Justicia y Género.

Marques-Pereira, B. (1993). Estado y Estado benefactor: metodologías feministas. Revista Mexicana de Sociología, 55(3), 121-131. doi: 10.2307/3540925.

Martínez-Franzoni, J. y Voorend, K. (2008). Transferencias condicionadas, regímenes de bienestar e igualdad de género: ¿blancos, negros o grises? Revista de Ciencias Sociales, (122), 115-131. Doi: 10.15517/RCS.V0I122.9879.

Molyneux, M. (2007). Change and Continuity in Social Protection in Latin America. Mothers at the service of the State? Switzerland: United Nations Research Institute for Social Development.

Oszlak, O. (Comp.) (1984). Teoría de la burocracia estatal: enfoques críticos. Buenos Aires: Paidós.

Paura, V. y Zibecchi, C. (2014). Dinámicas institucionales, lógicas de los actores y territorio en el estudio de la política social. Veinte años de investigación en la Argentina. En L. Pautassi (Dir.) Marginaciones sociales en el área metropolitana de Buenos Aires. Acceso a la justicia, capacidades estatales y movilización legal (pp. 333-396). Argentina: Biblos. 
Paura, V. y Zibecchi, C. (agosto, 2017). Mujeres actuando en los márgenes del Estado: aportes de los estudios, escenarios de reconocimiento y contextos de producción. En II Congreso Latinoamericano de Teoría Social y Teoría Política "Horizontes y dilemas del pensamiento contemporáneo en el sur global". Instituto de Altos Estudios Sociales (IDAES). Buenos Aires, Argentina.

Pautassi, L. y Zibecchi, C. (2010). La provisión de cuidado y la superación de la pobreza infantil. Programas de transferencias condicionadas en Argentina y el papel de las organizaciones sociales y comunitarias. Chile: CEPAL. Unicef.

Rodríguez-Enríquez, C. (2012). Políticas de atención a la pobreza y las desigualdades en América Latina: una revisión crítica desde la economía feminista. En V. Esquivel (Coord.) La economía feminista desde América Latina. Una hoja de ruta sobre los debates actuales en la región (pp. 390-437). Santo Domingo: ONU Mujeres.

Rodríguez-Gustá, A.L. (2014. Una misma política social, tres efectos de género disímiles: la implementación local de un programa de transferencia condicionada en la Argentina. Trabajo y Sociedad, 17(22), 559-576.

Schijman, E. y François-Lae, J. (2011): Las rondas de las mujeres por las ventanillas del Estado. Etnografía de un trabajo invisible. Trabajo y Sociedad, 15(16), 67-83.

Serrano, C. (2005). La política social en la globalización. Programas de protección en América Latina. Chile: CEPAL.

Skocpol, T. (1985). Bringing the State back in strategies of analysis in current research. Introducción. En B. Evans, D. Rueschemeyer y T. Skocpol (Comps.) Bringing the State Back in (pp, 3-43). Cambridge University Press.

Zibecchi, C. (2013). Trayectorias Asistidas. Un abordaje de los programas sociales en Argentina desde el enfoque de género. Argentina: EUDEBA.

Zibecchi, C. (2015). Cuidando en el territorio: El espacio comunitario como proveedor de cuidado. Argentina: Equipo Latinoamericano de justicia y de género.

Cómo citar:

Zibecchi, C. (2019). ¿Interlocutoras del Estado? El caso de las destinatarias de programas sociales y las referentes de organizaciones territoriales en la Argentina. Prospectiva. Revista de Trabajo Social e intervención social, (27), 29-54. Doi: 10.25100/prts.v0i27.7269. 